Service social

\title{
Protection de l'enfance - Réalité de l'intervention, par Robert Dubé et Marjolaine Saint-Jules, Montréal, Gaëtan Morin Éditeur, Fondation des enfants maltraités du Québec inc., 1987, 242 pages.
}

\section{Marcel Bonneau}

Volume 38, numéro 2-3, 1989

Les politiques sociales

URI : https://id.erudit.org/iderudit/706446ar

DOI : https://doi.org/10.7202/706446ar

Aller au sommaire du numéro

Éditeur(s)

École de service social de l'Université Laval

ISSN

1708-1734 (numérique)

Découvrir la revue

Citer ce compte rendu

Bonneau, M. (1989). Compte rendu de [Protection de l'enfance - Réalité de l'intervention, par Robert Dubé et Marjolaine Saint-Jules, Montréal, Gaëtan Morin Éditeur, Fondation des enfants maltraités du Québec inc., 1987, 242 pages.] Service social, 38(2-3), 319-321. https://doi.org/10.7202/706446ar d'utilisation que vous pouvez consulter en ligne. 
Létourneau est passé maître dans cet art de convaincre et de séduire, tout comme dans celui de la pédagogie. La singularité de cet ouvrage réside dans I'habileté de l'auteur à décortiquer le processus d'une technique ou d'une activité intellectuelle. Fort rigoureux dans sa démarche, il arrive à transmettre de façon simple, claire et précise, une matière qui pourrait être qualifiée d'aride et qui a fait l'objet de peu de publications. La démarche de l'auteur et la structure de son ouvrage suivent une logique sans faille; l'œuvre ne pourrait être plus systématique.

En définitive, il s'agit d'un excellent ouvrage de consultation qui regorge d'outils pratiques. Véritable exemple de travail méthodique, il est accessible au public auquel il s'adresse et même à l'autodidacte, ou encore à ceux et celles qui ne se sont pas astreints depuis un certain temps aux règles des travaux académiques.

Suzanne BÉRUBÉ

Étudiante à la maîtrise,

École de service social, Université Laval.

Protection de l'enfance - Réalité de l'intervention, par Robert Dubé et Marjolaine SAINT-JULES, Montréal, Gaëtan Morin Éditeur, Fondation des enfants maltraités du Québec inc., 1987, 242 pages.

Ce livre a pour objet la problématique des enfants maltraités et les systèmes de protection en place pour remédier aux insuffisances de leur milieu familial. Les trois sections du volume sont agencées de façon à faire cheminer le lecteur à travers une prise de conscience du problème, un regard sur les aspects dynamiques de l'intervention en protection de l'enfance et une vue d'ensemble des mesures de protection actuellement en place au Québec. Nous constatons une grande rigueur dans la démarche des auteurs, démarche basée sur une revue approfondie des écrits et une information très complète sur le sujet.

La première partie du volume intitulée "le problème ", présente la problématique sous différents angles. Ainsi, l'observation de l'enfant à travers I'histoire nous permet de saisir la considération qu'ont eue les humains à l'endroit de l'enfant et la place qui lui a été réservée au cours des siècles.

La définition et la recherche des causes de la maltraitance procurent au lecteur une occasion de bien circonscrire les abus physiques, la négligence et les abus sexuels. On y expose l'origine de ces problématiques et les facteurs explicatifs sous-jacents. Quant à la section portant sur les conséquences de la maltraitance, le lecteur ne pourra demeurer indifférent devant une telle présentation des séquelles et limites qui marqueront l'enfant victime, et ce, tout au cours de son développement. 
Un élément majeur semble ressortir de cette description de la problématique et il a trait à la violence vécue dans ces familles. En effet, tous ces gestes posés par des adultes envers les enfants sont souvent le fruit d'une violence vécue dans leur propre enfance ou vécue dans leur quotidien en réaction aux difficultés d'adaptation qu'éprouvent les adultes.

La seconde partie du volume porte sur "l'intervention de protection ». Les auteurs ont voulu présenter une section plus spécifique sur les façons d'intervenir auprès de ces jeunes en difficultés. Les principes d'intervention suggérés font ressortir des valeurs professionnelles axées sur le respect du client, du lien unissant l'enfant à ses parents et de la capacité du client à changer.

Les aspects évaluatifs et dynamiques de l'intervention préconisée apparaissent bien articulés et appropriés. Les modèles relationnels à développer dans l'intervention auprès des clients apportent une vision plus dynamique quant aux façons d'intervenir auprès de cette clientèle. II nous restera à voir, après l'application des conclusions du rapport Harvey sur la standardisation du fonctionnement des directions de la protection de la jeunesse, s'il sera encore aussi facile pour les intervenants de développer de telles relations avec les clients. Nous craignons que faute de temps, les intervenants ne puissent appliquer $d^{\prime}$ aussi beaux modèles $d^{\prime}$ 'intervention auprès de ces clientèles.

La dernière section du volume porte sur " les solutions "; or à ce niveau, les mesures de protection préconisées apparaissent des plus intéressantes. L'énumération se veut des plus complètes et fait appel à plusieurs personnes et organismes de toutes provenances. En effet, cette section présente bien le système de protection de la jeunesse, les articles de la loi et la façon de signaler toute situation pour laquelle une personne a des soupçons raisonnables de croire qu'un enfant est maltraité ou abusé.

Cependant, on parcourt trop rapidement les éléments ayant trait à la complémentarité des intervenants dans l'aide à dispenser à ces enfants et à leur famille. On observe dans la pratique quotidienne de plusieurs professionnels un modèle d'intervention centré sur leurs tâches spécifiques et peu de collaboration avec les autres intervenants. Le cloisonnement des pratiques professionnelles et l'impact qui en découle pour les services à ces clientèles ont été tenus sous silence par les auteurs.

La responsabilité de prise en charge des clientèles abusées ou négligées devient souvent la responsabilité quaxi exclusive du directeur de la protection de la jeunesse et de son personnel. À ce niveau, il y a des mentalités à changer, des façons de faire à modifier et il y a lieu d'inventer de nouveaux modèles d'aide.

Tout intervenant significatif ayant un lien avec ces familles dans le besoin devrait être mobilisé en faveur de l'intervention à mettre en place pour aider ces enfants victimes.

Je terminerai en soulignant que ce volume constitue un document de référence des plus pertinents quant à la définition et aux conséquences de la maltraitance. C'est une lecture que je recommande à tout intervenant qui débute dans le domaine ou qui désire réviser ses connaissances sur le sujet. 
Les pistes d'intervention proposées sont des plus intéressantes. Cependant, il aurait été très avantageux que les auteurs explorent plus à fond les modèles complémentaires de prise en charge de ces clientèles. Pour que ces jeunes traversent ces périodes de crise avec le moins de séquelles possible, la multidisciplinarité s'impose aux intervenants des milieux social, communautaire, médical et de l'éducation.

À qui incombe la protection de l'enfance maltraitée ? Ne serait-ce pas une responsabilité collective?

Marcel BONNEAU

Direction de la Protection de la jeunesse,

Sherbrooke.

Mouvement populaire et intervention communautaire de 1960 à nos jours, Continuités et ruptures, par Louis FAVREAU, Montréal, le Centre de formation populaire et les Éditions du Fleuve, Alternatives, 1989, 307 pages.

Le mouvement populaire et communautaire s'engage-t-il dans une période de déclin ou de mutation culturelle et sociale ? Voilà la question centrale qui est à l'origine d'une importante enquête qui a débuté en 1985 et qui demeure d'actualité en 1989.

Pour répondre à cette question, l'auteur nous invite à faire un peu $d$ 'histoire en abordant la dimension historique du mouvement populaire et communautaire québécois et ce pour les vingt-cinq dernières années. L'itinéraire qui nous est suggéré nous amène de la naissance des premiers comités de citoyens de la décennie 60 , en passant par les belles années du militantisme, les années d'incertitudes, de tâtonnements, pour conclure sur la possible relance de ce mouvement.

Le troisième chapitre nous fait pénétrer et sentir le riche vécu de trois quartiers populaires montréalais : Hochelaga-Maisonneuve, Centre-Sud et La Petite Patrie. À travers I'analyse de plusieurs groupes populaires, I'auteur cherche à dégager des constantes concernant le démarrage, la survie et la consolidation de ces groupes. Ces quartiers ont subi et continuent de vivre une mutation sociale très importante; alors qu'hier la culture de pauvreté était très présente, aujourd'hui elle se fait envahir par une nouvelle mentalité qui peut se caractériser par une capacité de transformer les problèmes en projets.

Ce volume aborde aussi avec beaucoup de justesse et d'honnêteté la difficile question de l'implication du mouvement populaire et communautaire dans l'économie et plus précisément dans la création d'emplois et d'entreprises. La récession du début des années ' 80 a bouleversé bien des valeurs. Le mouvement populaire a un nouveau visage. D'un côté, l'implication des militants et militantes sur le terrain de l'économie est une réalité bien présente; de l'autre côté, les groupes de services et de pression demeurent et continuent d'exercer une forme de pouvoir non négligeable. Dans ce chapitre, 